Sequential Anal. Vol. 29, No. 3, 2010, (311-327)

Research Report No. 5, 2009, Probab. Statist. Group Manchester (17 pp)

Dedicated to Albert N. Shiryaev on the occasion of his 75 th birthday

\title{
The British Asian Option
}

\author{
K. Glover, G. Peskir \& F. Samee
}

\begin{abstract}
Following the economic rationale of [7] and [8] we present a new class of Asian options where the holder enjoys the early exercise feature of American options whereupon his payoff (deliverable immediately) is the 'best prediction' of the European payoff under the hypothesis that the true drift of the stock price equals a contract drift. Inherent in this is a protection feature which is key to the British Asian option. Should the option holder believe the true drift of the stock price to be unfavourable (based upon the observed price movements) he can substitute the true drift with the contract drift and minimise his losses. The practical implications of this protection feature are most remarkable as not only is the option holder afforded a unique protection against unfavourable stock price movements (covering the ability to sell in a liquid option market completely endogenously) but also when the stock price movements are favourable he will generally receive high returns. We derive a closed form expression for the arbitrage-free price in terms of the rational exercise boundary and show that the rational exercise boundary itself can be characterised as the unique solution to a nonlinear integral equation. Using these results we perform a financial analysis of the British Asian option that leads to the conclusions above and shows that with the contract drift properly selected the British Asian option becomes a very attractive alternative to the classic (European) Asian option.
\end{abstract}

\section{Introduction}

The purpose of the present paper is to introduce and examine the British payoff mechanism (see [7] and [8]) in the context of Asian options. There are various types of Asian options that may be considered to this end: (i) calls and puts; (ii) those with fixed or floating strike; (iii) the average may be arithmetic or geometric; (iv) the weighting in the average may be equal or flexible (for more details on each of these types see e.g. [2] and the references therein). This leads to an extensive programme of research that we open in this paper by focusing on a pure (arithmetic) average Asian option (with no strike) that plays a canonical role among all other possibilities and has the advantage of (relative) tractability.

Following the economic rationale of [7] and [8] we thus introduce a new Asian option which endogenously provides its holder with a protection mechanism against unfavourable stock price movements. This mechanism is intrinsically built into the option contract using the concept of optimal prediction (see e.g. [1] and the references therein) and we refer to such contracts as 'British' for the reasons outlined in [7] and [8] where the British put and call options were

Mathematics Subject Classification 2000. Primary 91B28, 60G40. Secondary 35R35, 45G10, 60J60.

Key words and phrases: British Asian option, American Asian option, European Asian option, fixed/floating strike, arithmetic/geometric average, flexible Asian options, arbitrage-free price, rational exercise boundary, liquid/illiquid market, geometric Brownian motion, the Shiryaev process, optimal stopping, parabolic freeboundary problem, nonlinear integral equation, local time-space calculus. 
introduced. Similarly to [7] and [8] most remarkable about the British Asian option is not only that it provides a unique protection against unfavourable stock price movements (endogenously covering the ability of a European Asian holder to sell his contract in a liquid option market) but also when the stock price movements are favourable it enables its holder to obtain high returns. This reaffirms the fact noted in [7] and [8] that the British feature of optimal prediction acts as a powerful tool for generating financial instruments (in the context of Asian options) which aim at both providing protection against unfavourable price movements as well as securing high returns when these movements are favourable. We recall that these combined features appear to be especially appealing as they address problems of liquidity and return completely endogenously (reducing the need for exogenous regulation).

The paper is organised as follows. In Section 2 we present a basic motivation for the British Asian option. In Section 3 we formally define the British Asian option and present some of its basic properties. This is continued in Section 4 where we derive a closed form expression for the arbitrage-free price in terms of the rational exercise boundary (the early-exercise premium representation) and show that the rational exercise boundary itself can be characterised as the unique solution to a nonlinear integral equation (Theorem 1). Using these results in Section 5 we present a financial analysis of the British Asian option (making comparisons with the European Asian option). This analysis provides more detail/insight into the full scope of the conclusions briefly outlined above. We conclude in Section 6 by presenting the other British Asian options addressed in (i)-(iv) above and providing an agenda for future research.

\section{Basic motivation for the British Asian option}

The basic economic motivation for the British Asian option is parallel to that of the British put and call options (see [7] and [8]). In this section we briefly review key elements of this motivation by focusing on a pure (arithmetic) average Asian option (with no strike) that plays a canonical role among all other possibilities and has the advantage of (relative) tractability. We remark that the full financial scope of the British Asian option goes beyond these initial considerations (see Sections 5 and 6 below for further details).

1. Consider the financial market consisting of a risky stock $S$ and a riskless bond $B$ whose prices respectively evolve as

$$
\begin{aligned}
& d S_{t}=\mu S_{t} d t+\sigma S_{t} d W_{t} \quad\left(S_{0}=s\right) \\
& d B_{t}=r B_{t} d t \quad\left(B_{0}=1\right)
\end{aligned}
$$

where $\mu \in \mathbb{R}$ is the appreciation rate (drift), $\sigma>0$ is the volatility coefficient, $W=\left(W_{t}\right)_{t \geq 0}$ is a standard Wiener process defined on a probability space $(\Omega, \mathcal{F}, \mathrm{P})$, and $r>0$ is the interest rate. Recall that an arithmetic average Asian option of European type (with no strike) is a financial contract between a seller/hedger and a buyer/holder entitling the latter to exercise the option at a specified maturity time $T>0$ and receive the payoff

$$
A_{T}=\frac{1}{T} \int_{0}^{T} S_{t} d t
$$

from the seller. Standard hedging arguments based on self-financing portfolios imply that the arbitrage-free price of the option is given by

$$
V=\widetilde{\mathrm{E}}\left[\mathrm{e}^{-r T} A_{T}\right]
$$


where the expectation $\tilde{\mathrm{E}}$ is taken with respect to the (unique) equivalent martingale measure $\tilde{P}$ (see e.g. [11]). In this section (as in [7] and [8]) we will analyse the option from the standpoint of a true buyer. By 'true buyer' we mean a buyer who has no ability or desire to sell the option nor to hedge his own position. Thus every true buyer will exercise the option at time $T$ in accordance with the rational performance. For more details on the motivation and interest for considering a true buyer in this context see [7].

2. With this in mind we now return to the Asian holder whose payoff is given by (2.3) above. Recall that the unique strong solution to (2.1) is given by

$$
S_{t}=S_{t}(\mu)=s \exp \left(\sigma W_{t}+\left(\mu-\frac{\sigma^{2}}{2}\right) t\right)
$$

under $\mathrm{P}$ for $t \in[0, T]$ where $\mu \in \mathbb{R}$ is the actual drift. Inserting (2.5) into (2.3) we find that the expected value of the buyer's payoff equals

$$
P=P(\mu)=\mathrm{E}\left[\mathrm{e}^{-r T} A_{T}\right]=\frac{s}{\mu T}\left(\mathrm{e}^{(\mu-r) T}-\mathrm{e}^{-r T}\right) .
$$

Moreover, it is well known that $\operatorname{Law}(S(\mu) \mid \tilde{\mathrm{P}})$ is the same as $\operatorname{Law}(S(r) \mid \mathrm{P})$ so that the arbitrage-free price of the option equals

$$
V=P(r)=\frac{s}{r T}\left(1-\mathrm{e}^{-r T}\right) .
$$

A direct comparison of (2.6) and (2.7) shows that if $\mu=r$ then the return is 'fair' for the buyer, in the sense that $V=P$, where $V$ represents the value of his investment and $P$ represents the expected value of his payoff. On the other hand, if $\mu>r$ then the return is 'favourable' for the buyer, in the sense that $V<P$, and if $\mu<r$ then the return is 'unfavourable' for the buyer, in the sense that $V>P$ with the same interpretations as above. We recall that the actual drift $\mu$ is unknown at time $t=0$ and also difficult to estimate at later times $t \in(0, T]$ unless $T$ is unrealistically large.

3. The brief analysis above shows that whilst the actual drift $\mu$ of the underlying stock price is irrelevant in determining the arbitrage-free price of the option, to a (true) buyer it is crucial, and he will buy the option if he believes that $\mu>r$. If this turns out to be the case then on average he will make a profit. Thus, after purchasing the option, the Asian holder will be happy if the observed stock price movements reaffirm his belief that $\mu>r$.

The British Asian option seeks to address the opposite scenario: What if the option holder observes stock price movements which change his belief regarding the actual drift and cause him to believe that $\mu<r$ instead? In this contingency the British Asian holder is effectively able to substitute this unfavourable drift with a contract drift and minimise his losses. In this way he is endogenously protected from any stock price drift smaller than the contract drift. The value of the contract drift is therefore selected to represent the buyer's level of tolerance for the deviation of the actual drift from his original belief.

It will be shown below (similarly to [7] and [8]) that the practical implications of this protection feature are most remarkable as not only is the option holder afforded a unique protection against unfavourable stock price movements (covering the ability to sell in a liquid option market completely endogenously) but also when the stock price movements are favourable he will generally receive high returns. We refer to the final paragraph of Section 2 in [8] for further comments regarding the option holder's ability to sell his contract (releasing the true buyer's 
perspective) and its connection with option market liquidity. This translates into the present setting without changes.

\section{The British Asian option: Definition and basic properties}

We begin this section by presenting a formal definition of the British Asian option. This is then followed by a brief analysis of the optimal stopping problem and the free-boundary problem characterising the arbitrage-free price and the rational exercise strategy. These considerations are continued in Sections 4 and 5 below.

1. Consider the financial market consisting of a risky stock $S$ and a riskless bond $B$ whose prices evolve as (2.1) and (2.2) respectively, where $\mu \in \mathbb{R}$ is the appreciation rate (drift), $\sigma>0$ is the volatility coefficient, $W=\left(W_{t}\right)_{t \geq 0}$ is a standard Wiener process defined on a probability space $(\Omega, \mathcal{F}, \mathrm{P})$, and $r>0$ is the interest rate. Let a maturity time $T>0$ be given and fixed, and let $A_{T}$ denote the average stock price given by (2.3) above.

Definition 1. The British Asian option is a financial contract between a seller/hedger and a buyer/holder entitling the latter to exercise at any (stopping) time $\tau$ prior to $T$ whereupon his payoff (deliverable immediately) is the 'best prediction' of the European payoff $A_{T}$ given all the information up to time $\tau$ under the hypothesis that the true drift of the stock price equals $\mu_{c}$.

The quantity $\mu_{c}$ is defined in the option contract and we refer to it as the 'contract drift'. We will show below that the contract drift must satisfy

$$
\mu_{c}<0
$$

since otherwise it would be rational to exercise immediately (i.e. the buyer would be overprotected). Recall from Section 2 above that the value of the contract drift is selected to represent the buyer's level of tolerance for the deviation of the true drift $\mu$ from his original belief. It will be shown in Section 5 below that this protection feature has remarkable implications both in terms of liquidity and return.

2. Denoting by $\left(\mathcal{F}_{t}\right)_{0 \leq t \leq T}$ the natural filtration generated by $S$ (possibly augmented by null sets or in some other way of interest) the payoff of the British Asian option at a given stopping time $\tau$ with values in $[0, T]$ can be formally written as

$$
\mathrm{E}^{\mu_{c}}\left(A_{T} \mid \mathcal{F}_{\tau}\right)
$$

where the conditional expectation is taken with respect to a new probability measure $\mathrm{P}^{\mu_{c}}$ under which the stock price $S$ evolves as

$$
d S_{t}=\mu_{c} S_{t} d t+\sigma S_{t} d W_{t}
$$

with $S_{0}=s$ in $(0, \infty)$. Comparing (2.1) and (3.3) we see that the effect of exercising the British Asian option is to substitute the true (unknown) drift of the stock price with the contract drift for the remaining term of the contract. 
3. Setting $I_{t}=\int_{0}^{t} S_{s} d s$ for $t \in[0, T]$ and using stationary and independent increments of $W$ governing $S$ we find that

$$
\begin{aligned}
\mathrm{E}^{\mu_{c}}\left(A_{T} \mid \mathcal{F}_{t}\right) & =\mathrm{E}^{\mu_{c}}\left(\frac{1}{T} \int_{0}^{T} S_{v} d v \mid \mathcal{F}_{t}\right)=\frac{1}{T} \mathrm{E}^{\mu_{c}}\left(I_{t}+\int_{t}^{T} S_{v} d v \mid \mathcal{F}_{t}\right) \\
& =\frac{1}{T}\left[I_{t}+S_{t} \mathrm{E}^{\mu_{c}}\left(\int_{t}^{T} \frac{S_{v}}{S_{t}} d v \mid \mathcal{F}_{t}\right)\right]=\frac{1}{T}\left[I_{t}+S_{t} \mathrm{E}^{\mu_{c}}\left(\int_{0}^{T-t} \frac{1}{s} S_{v} d v\right)\right] \\
& =\frac{1}{T}\left[I_{t}+S_{t} \int_{0}^{T-t} \mathrm{e}^{\mu_{c} v} d v\right]=\frac{1}{T}\left[I_{t}+S_{t} \frac{1}{\mu_{c}}\left(\mathrm{e}^{\mu_{c}(T-t)}-1\right)\right]
\end{aligned}
$$

for $t \in[0, T]$. Standard hedging arguments based on self-financing portfolios (with consumption) imply that the arbitrage-free price of the British Asian option is given by

$$
V=\sup _{0 \leq \tau \leq T} \widetilde{\mathrm{E}}\left[\mathrm{e}^{-r \tau} \mathrm{E}^{\mu_{c}}\left(A_{T} \mid \mathcal{F}_{\tau}\right)\right]
$$

where the supremum is taken over all stopping times $\tau$ of $S$ with values in $[0, T]$ and $\tilde{\mathrm{E}}$ is taken with respect to the (unique) equivalent martingale measure $\tilde{P}$. From (3.4) we see that the underlying Markov process in the optimal stopping problem (3.5) equals $\left(t, I_{t}, S_{t}\right)$ for $t \in[0, T]$ thus making it three-dimensional.

4. Since $\operatorname{Law}(S(\mu) \mid \tilde{\mathrm{P}})$ is the same as $\operatorname{Law}(S(r) \mid \mathrm{P})$, it follows from the well-known additive structure of $I$ and multiplicative structure of $S$ that (3.5) extends as follows

$$
V(t, i, s)=\sup _{0 \leq \tau \leq T-t} \mathrm{E}\left[\frac{\mathrm{e}^{-r \tau}}{T}\left(i+\int_{0}^{\tau} s S_{v} d v+s S_{\tau} \frac{1}{\mu_{c}}\left(\mathrm{e}^{\mu_{c}(T-t-\tau)}-1\right)\right)\right]
$$

for $t \in[0, T], i \in[0, \infty)$ and $s \in(0, \infty)$ where the supremum is taken as in (3.5) above and the process $S=S(r)$ under $\mathrm{P}$ solves

$$
d S_{t}=r S_{t} d t+\sigma S_{t} d W_{t}
$$

with $S_{0}=1$. By the Girsanov theorem it follows that

$$
\mathrm{E}\left[\frac{\mathrm{e}^{-r \tau}}{T}\left(i+\int_{0}^{\tau} s S_{v} d v+s S_{\tau} \frac{1}{\mu_{c}}\left(\mathrm{e}^{\mu_{c}(T-t-\tau)}-1\right)\right)\right]=s \widehat{\mathrm{E}}\left[\frac{1}{T}\left(X_{\tau}^{x}+\frac{1}{\mu_{c}}\left(\mathrm{e}^{\mu_{c}(T-t-\tau)}-1\right)\right)\right]
$$

where as in [4] (see also [3, p. 377]) we set

$$
X_{t}^{x}=\frac{i+\int_{0}^{t} s S_{v} d v}{s S_{t}}=\frac{x+I_{t}}{S_{t}}
$$

with $x=i / s$ and $\hat{\mathrm{P}}$ is defined by $d \hat{\mathrm{P}}=\exp \left(\sigma W_{T}-\left(\sigma^{2} / 2\right) T\right) d \mathrm{P}$ so that $\hat{W}_{t}=W_{t}-\sigma t$ is a standard Wiener process under $\hat{\mathrm{P}}$ for $t \in[0, T]$. By Itô's formula one finds that

$$
d X_{t}=\left(1-r X_{t}\right) d t+\sigma X_{t} d \widetilde{W_{t}} \quad\left(X_{0}=x\right)
$$

under $\hat{\mathrm{P}}$ where $\tilde{W}=-\hat{W}$ is a standard Wiener process and $x \in[0, \infty)$. The Markov process $X$ is called the Shiryaev process (see [6] for more details). Its state space equals $[0, \infty$ ) where 0 is an entrance boundary point. Setting

$$
G^{\mu_{c}}(t, x)=\frac{1}{T}\left(x+\frac{1}{\mu_{c}}\left(\mathrm{e}^{\mu_{c}(T-t)}-1\right)\right)
$$


consider the optimal stopping problem

$$
V(t, x)=\sup _{0 \leq \tau \leq T-t} \mathrm{E}\left[G^{\mu_{c}}\left(t+\tau, X_{\tau}^{x}\right)\right]
$$

for $t \in[0, T]$ and $x \in[0, \infty)$ where the supremum is taken over all stopping times $\tau$ of $X$ with values in $[0, T-t]$ and $\mathrm{E}$ stands for $\hat{\mathrm{E}}$ to simplify the notation. It follows from (3.6) and (3.8) using (3.11) and (3.12) that

$$
V(t, i, s)=s V\left(t, \frac{i}{s}\right)
$$

for $t \in[0, T], i \in[0, \infty)$ and $s \in(0, \infty)$. In this way we have reduced the three-dimensional problem (3.6) to the two-dimensional problem (3.12). Using the established probabilistic techniques (see e.g. [10]) one can verify that the value function $V:[0, T] \times[0, \infty) \rightarrow \mathbb{R}$ is continuous and the stopping time $\tau_{D}=\inf \left\{s \in[0, T-t] \mid\left(t+s, X_{s}^{x}\right) \in D\right\}$ is optimal in (3.12) for $(t, x) \in[0, T) \times[0, \infty)$ given and fixed, where $C=\left\{V>G^{\mu_{c}}\right\}$ is the continuation set and $D=\left\{V=G^{\mu_{c}}\right\}$ is the stopping set in $[0, T] \times[0, \infty)$.

5. To gain a deeper insight into the solution to the optimal stopping problem (3.12), let us note that Itô's formula and the optional sampling theorem yield

$$
\mathrm{E}\left[G^{\mu_{c}}\left(t+\tau, X_{\tau}^{x}\right)\right]=G^{\mu_{c}}(t, x)+\mathrm{E}\left[\int_{0}^{\tau} H^{\mu_{c}}\left(t+s, X_{s}^{x}\right) d s\right]
$$

for all stopping times $\tau$ of $X$ with values in $[0, T-t]$ with $t \in[0, T)$ and $x \in[0, \infty)$ given and fixed, where the function $H^{\mu_{c}}$ is given by

$$
H^{\mu_{c}}(t, x)=G_{t}^{\mu_{c}}+(1-r x) G_{x}^{\mu_{c}}+\frac{\sigma^{2}}{2} x^{2} G_{x x}^{\mu_{c}}=\frac{1}{T}\left(1-r x-\mathrm{e}^{\mu_{c}(T-t)}\right) .
$$

From (3.14) and (3.15) we see that if $\mu_{c} \geq 0$ then $H^{\mu_{c}} \leq 0$ so that it is optimal to stop at once in (3.12) as claimed in (3.1) above. If $\mu_{c}<0$ then the function $h:[0, T] \rightarrow \mathbb{R}$ solving $H^{\mu_{c}}(t, h(t))=0$ is given by

$$
h(t)=\frac{1}{r}\left(1-\mathrm{e}^{\mu_{c}(T-t)}\right)
$$

for $t \in[0, T]$. In this case it is evident that $H^{\mu_{c}}(t, x)>0$ for $x \in[0, h(t))$ and $H^{\mu_{c}}(t, x)<0$ for $x \in(h(t), \infty)$ when $t \in[0, T)$ is given and fixed. In view of (3.14) this implies that no point $(t, x)$ in $[0, T) \times(0, \infty)$ with $x<h(t)$ is a stopping point (for this one can make use of the first exit time from a sufficiently small time-space ball centred at the point). Likewise, it is also clear and can be verified that if $x>h(t)$ and $t<T$ is sufficiently close to $T$ then it is optimal to stop immediately (since the gain obtained from being below $h$ cannot offset the cost of getting there due to the lack of time). This shows that the optimal stopping boundary $b:[0, T] \rightarrow[0, \infty]$ separating the continuation set from the stopping set satisfies $b(T)=h(T)$ and this value equals 0 . Moreover, noting that $x \mapsto H(t, x)$ is decreasing on $[0, \infty)$ for every $t \in[0, T]$, it follows that the continuation set is given by $C=\{(t, x) \in[0, T) \times[0, \infty) \mid$ $x<b(t)\}$ and the stopping set is given by $D=\{(t, x) \in[0, T] \times[0, \infty) \mid x \geq b(t)\}$ so that the optimal stopping time in (3.12) is given by

$$
\tau_{b}=\inf \left\{t \in[0, T] \mid X_{t} \geq b(t)\right\} .
$$




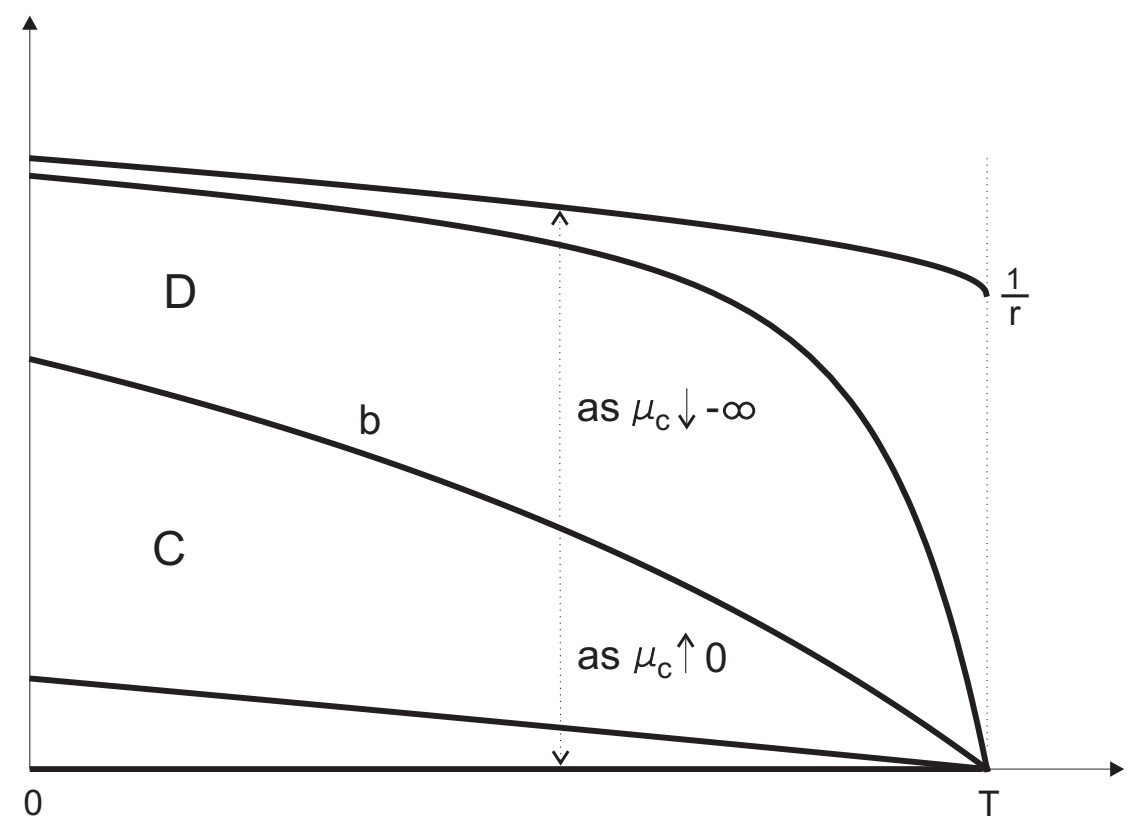

Figure 1. A computer drawing showing how the rational exercise boundary of the British Asian option changes as one varies the contract drift.

Finally, noting that $t \mapsto H(t, x)$ is decreasing on $[0, T]$ for every $x \in[0, \infty)$, it follows that $t \mapsto b(t)$ is decreasing on $[0, T]$. It is also clear and can be verified that if the initial point $x>0$ of the process $X$ is sufficiently large then it is optimal to stop immediately (since the gain obtained from being below $h$ cannot offset the cost of getting there due to the shortage of time). This shows that the optimal stopping boundary $b$ is finite valued. Using the established probabilistic techniques (see e.g. [10]) one can also verify that $V$ satisfies the smooth fit property at $b$ (in the sense that $x \mapsto V(t, x)$ is $C^{1}$ at $b(t)$ for every $t \in[0, T]$ ) and $b:[0, T] \rightarrow[0, \infty)$ is continuous. The stopping time (3.17) represents the rational exercise strategy for the British Asian option and plays a key role in financial analysis of the option.

6. Standard Markovian arguments lead to the following free-boundary problem (for the value function $V=V(t, x)$ and the optimal stopping boundary $b=b(t)$ to be determined):

$$
\begin{aligned}
& V_{t}+(1-r x) V_{x}+\frac{\sigma^{2}}{2} x^{2} V_{x x}=0 \text { for } x \in(0, b(t)) \text { and } t \in[0, T) \\
& V(t, b(t))=\frac{1}{T}\left(b(t)+\frac{1}{\mu_{c}}\left(\mathrm{e}^{\mu_{c}(T-t)}-1\right)\right) \text { for } t \in[0, T] \quad \text { (stopping) } \\
& V_{x}(t, b(t))=\frac{1}{T} \text { for } t \in[0, T] \quad \text { (smooth fit) } \\
& V_{t}(t, 0+)+V_{x}(t, 0+)=0 \text { for } t \in[0, T] \quad \text { (entrance boundary) }
\end{aligned}
$$

where $b(T)=0$ and we set $V(t, x)=G^{\mu_{c}}(t, x)$ for $x \geq b(t)$ and $t \in[0, T]$ (see e.g. [10]). The arguments outlined above combined with those given in the proof of Theorem 1 below show that this free-boundary problem has a unique solution $V$ and $b$ which coincide with the value function (3.12) and the optimal stopping boundary respectively. 
Note that $t \mapsto V(t, x)$ is decreasing on $[0, T]$ for every $x \in[0, \infty)$ and $x \mapsto V(t, x)$ is increasing and convex on $[0, \infty)$ for every $t \in[0, T]$ (since $G^{\mu_{c}}$ is so in both cases). Note also that if we let $\mu_{c}$ run from 0 to $-\infty$ then the optimal stopping boundary $b$ moves from the zero function to a continuous decreasing function $b_{-\infty}:[0, T] \rightarrow \mathbb{R}$ satisfying $b_{-\infty}(T)=1 / r$ (see Figure 1$)$. The limiting boundary $b_{-\infty}$ is optimal in the problem (3.12) where $G^{-\infty}(t, x)=x / T$ for $(t, x) \in[0, T] \times[0, \infty)$. This problem corresponds to the integral option (of American type) where the conditional expectation from (3.2) is replaced by $I_{\tau} / T$ in (3.5). We will see in Section 5 below that fuller understanding of the different positions of the optimal stopping boundary is important for a correct/desired choice of the contract drift $\mu_{c}$ in relation to other parameters in the British Asian option.

7. We conclude this section with a few remarks on the volatility parameter in the British payoff mechanism. Unlike the British put and call options (cf. [7] and [8]) the volatility parameter $\sigma$ is not present in the payoff (3.11) for this zero-strike variant of the British Asian option. It should be noted, however, that for any of the non-zero-strike variants defined in Section 6 below, the volatility parameter will inevitably become present in the corresponding British payoff. Considering the latter from a practical perspective, a natural question that arises is what value of the parameter $\sigma$ should be used in the contract specification/payoff? Both counterparties to the option trade must agree on the value of this parameter, or at least agree on how it should be calculated from future market observables, at the initiation of the contract. However, whilst this question has many practical implications, it does not pose any conceptual difficulties under the current modelling framework. Since the underlying process is assumed to be a geometric Brownian motion, the (constant) volatility of the stock price is effectively known by all market participants, since one may take any of the standard estimators for the volatility over an arbitrarily small time period prior to the initiation of the contract. This is in direct contrast to the situation with stock price drift $\mu$, whose value/form is inherently unknown, nor can be reasonably estimated (without an impractical amount of data), at the initiation of the contract. In this sense, it seems natural to provide a true buyer with protection from an ambiguous drift rather than a 'known' volatility, at least in this canonical Black-Scholes setting. We remark however that as soon as one departs from the current modelling framework, and gets closer to a more practical perspective, it is clear that the specification of the contract volatility may indeed become very important. In this wider framework one is naturally led to consider the relationship between the realised/implied volatility and the 'contract volatility' using the same/similar rationale as for the actual drift and the 'contract drift' in the text above. Due to the fundamental difference between the drift and the volatility in the canonical (Black-Scholes) setting, and the highly applied nature of such modelling issues, these features of the British payoff mechanism are left as the subject of future development.

\section{The arbitrage-free price and the rational exercise boundary}

In this section we derive a closed form expression for the arbitrage-free price $V$ in terms of the rational exercise boundary $b$ (the early-exercise premium representation) and show that the rational exercise boundary $b$ itself can be characterised as the unique solution to a nonlinear integral equation (Theorem 1). 
We will make use of the following function in Theorem 1 below:

$$
K(t, x, v, y)=\int_{0}^{\infty} \int_{0}^{\infty} \frac{1}{T}\left(\mathrm{e}^{\mu_{c}(T-v)}+r \frac{x+i}{s}-1\right) I\left(\frac{x+i}{s}>y\right) f(v-t, i, s) d i d s
$$

for $t \in[0, T), x \geq 0, v \in(t, T)$ and $y \geq 0$, where $(i, s) \mapsto f(u, i, s)$ is the probability density function of $\left(I_{u}, S_{u}\right)$ under $\hat{\mathrm{P}}$ with $S_{0}=1$ given by

$$
\begin{aligned}
f(u, i, s)= & \frac{2 \sqrt{2}}{\pi^{3 / 2} \sigma^{3}} \frac{s^{r / \sigma^{2}}}{i^{2} \sqrt{u}} \exp \left(\frac{2 \pi^{2}}{\sigma^{2} u}-\frac{\left(r+\sigma^{2} / 2\right)^{2}}{2 \sigma^{2}} u-\frac{2}{\sigma^{2} i}(1+s)\right) \\
& \times \int_{0}^{\infty} \exp \left(-\frac{2 z^{2}}{\sigma^{2} u}-\frac{4 \sqrt{s}}{\sigma^{2} i} \cosh (z)\right) \sinh (z) \sin \left(\frac{4 \pi z}{\sigma^{2} u}\right) d z
\end{aligned}
$$

for $i>0$ and $s>0$ where $u=v-t>0$. It should be noted that $K(t, x, v, b(v))>0$ for all $t \in[0, T), x \geq 0$ and $v \in(t, T)$ since the term in front of the indicator function in the double integral equals $-H^{\mu_{c}}\left(v, \frac{x+i}{s}\right)$ and $H^{\mu_{c}}(v, z)<0$ for all $z>b(v)$ because $b$ lies above $h$ (recall (3.16) above). For a derivation of the right-hand side in (4.2) using the result in [12, p. 527] see e.g. the Appendix in [9].

The main result of this section may now be stated as follows.

Theorem 1. The arbitrage-free price of the British Asian option (with $s=1$ and $i=x$ in (3.13) above) admits the following early-exercise premium representation

$$
V(t, x)=\frac{1}{T}\left(x e^{-r(T-t)}+\frac{1}{r}\left(1-e^{-r(T-t)}\right)\right)+\int_{t}^{T} K(t, x, v, b(v)) d v
$$

for all $(t, x) \in[0, T] \times[0, \infty)$, where the first term is the arbitrage-free price of the European Asian option and the second term is the early-exercise premium. (If $s \neq 1$ then (4.3) with $x=i / s$ needs to be inserted in (3.13) above.)

The rational exercise boundary of the British Asian option can be characterised as the unique continuous solution $b:[0, T] \rightarrow \mathbb{R}_{+}$to the nonlinear integral equation

$$
\frac{1}{T}\left(b(t)\left(1-e^{-r(T-t)}\right)+\frac{1}{\mu_{c}}\left(e^{\mu_{c}(T-t)}-1\right)-\frac{1}{r}\left(1-e^{-r(T-t)}\right)\right)=\int_{t}^{T} K(t, b(t), v, b(v)) d v
$$

satisfying $b(t) \geq h(t)$ for all $t \in[0, T]$ where $h$ is defined by (3.16) above.

Proof. We first derive (4.3) and show that the rational exercise boundary solves (4.4). We then show that (4.4) cannot have other (continuous) solutions. The proof of these facts is similar to the proof of Theorem 1 in [8] and we present all details for completeness.

1. Recall that the value function $V:[0, T] \times[0, \infty) \rightarrow \mathbb{R}$ and the rational exercise boundary $b:[0, T] \rightarrow \mathbb{R}_{+}$solve the free-boundary problem (3.18)-(3.21) (where $V$ extends as $G^{\mu_{c}}$ above $b)$, set $C_{b}=\{(t, x) \in[0, T) \times[0, \infty) \mid x<b(t)\}$ and $D_{b}=\{(t, x) \in[0, T) \times[0, \infty) \mid x>b(t)\}$, and let $\mathbb{L}_{X} V(t, x)=(1-r x) V_{x}(t, x)+\frac{\sigma^{2}}{2} x^{2} V_{x x}(t, x)$ for $(t, x) \in C_{b} \cup D_{b}$. Then $V$ and $b$ are continuous functions satisfying the following conditions: (i) $V$ is $C^{1,2}$ on $C_{b} \cup D_{b}$; (ii) $b$ is of bounded variation (decreasing); (iii) $\mathrm{P}\left(X_{t}^{x}=c\right)=0$ for all $c>0$ whenever $t \in[0, T]$ and 
$x \geq 0$; (iv) $V_{t}+\mathbb{L}_{X} V$ is locally bounded on $C_{b} \cup D_{b}$ (recall that $V$ satisfies (3.18) on $C_{b}$ and coincides with $G^{\mu_{c}}$ on $\left.D_{b}\right)$; (v) $x \mapsto V(t, x)$ is convex on $[0, \infty)$ for every $t \in[0, T]$; and (vi) $t \mapsto V_{x}(t, b(t) \pm)=G_{x}^{\mu_{c}}(t, b(t))=1 / T$ is continuous on $[0, T]$ (recall that $V$ satisfies the smooth-fit condition (3.20) at $b)$. From these conditions we see that the local time-space formula [5] is applicable to $(s, y) \mapsto V(t+s, y)$ with $t \in[0, T)$ given and fixed. Fixing also an arbitrary $x \geq 0$ this yields

$$
\begin{aligned}
V(t & \left.+s, X_{s}^{x}\right)=V(t, x) \\
& +\int_{0}^{s}\left(V_{t}+\mathbb{L}_{X} V\right)\left(t+v, X_{v}^{x}\right) I\left(X_{v}^{x} \neq b(t+v)\right) d v+M_{s}^{b} \\
& +\frac{1}{2} \int_{0}^{s}\left(V_{x}\left(t+v, X_{v}^{x}+\right)-V_{x}\left(t+v, X_{v}^{x}-\right)\right) I\left(X_{v}^{x}=b(t+v)\right) d \ell_{v}^{b}\left(X^{x}\right)
\end{aligned}
$$

where $M_{s}^{b}=\sigma \int_{0}^{s} X_{v}^{x} V_{x}\left(t+v, X_{v}^{x}\right) I\left(X_{v}^{x} \neq b(t+v)\right) d W_{v}$ is a continuous local martingale for $s \in[0, T-t]$ and $\ell^{b}\left(X^{x}\right)=\left(\ell_{v}^{b}\left(X^{x}\right)\right)_{0 \leq v \leq s}$ is the local time of $X^{x}=\left(X_{v}^{x}\right)_{0 \leq v \leq s}$ on the curve $b$ for $s \in[0, T-t]$. Moreover, since $V$ satisfies (3.18) on $C_{b}$ and equals $G^{\mu_{c}}$ on $D_{b}$, and the smooth-fit condition (3.20) holds at $b$, we see that (4.5) simplifies to

$$
V\left(t+s, X_{s}^{x}\right)=V(t, x)+\int_{0}^{s} H^{\mu_{c}}\left(t+v, X_{v}^{x}\right) I\left(X_{v}^{x}>b(t+v)\right) d v+M_{s}^{b}
$$

for $s \in[0, T-t]$ and $(t, x) \in[0, T) \times[0, \infty)$.

2. We show that $M^{b}=\left(M_{s}^{b}\right)_{0 \leq s \leq T-t}$ is a martingale for $t \in[0, T)$. For this, note that since $x \mapsto V(t, x)$ is increasing and convex, it follows that

$$
0 \leq V_{x}(t, x) \leq G_{x}^{\mu_{c}}(t, b(t))=1 / T
$$

for all $t \in[0, T)$ and all $x \geq 0$ (recall that $V$ equals $G^{\mu_{c}}$ above $b$ ). Hence by Jensen's inequality we find that

$$
\begin{aligned}
\mathrm{E}\left\langle M^{b}, M^{b}\right\rangle_{T-t}^{1 / 2} & =\sigma \mathrm{E}\left(\int_{0}^{T-t}\left(X_{v}^{x}\right)^{2} V_{x}^{2}\left(t+v, X_{v}^{x}\right) I\left(X_{v}^{x} \neq b(t+v)\right) d v\right)^{1 / 2} \\
& \leq \frac{\sigma}{T} \frac{1}{\sqrt{T-t}} \int_{0}^{T-t} \mathrm{E} X_{v}^{x} d v<\infty
\end{aligned}
$$

where the final conclusion follows from the identity

$$
\mathrm{E} X_{v}^{x}=x \mathrm{e}^{-r v}+\frac{1}{r}\left(1-\mathrm{e}^{-r v}\right)
$$

which is readily derived from (3.10) above. Hence $M^{b}$ is a martingale as claimed.

3. Replacing $s$ by $T-t$ in (4.6), using that $V(T, x)=G^{\mu_{c}}(T, x)$ for $x \geq 0$, taking $\mathrm{E}$ on both sides and applying the optional sampling theorem, we get

$$
\begin{aligned}
\mathrm{E} G^{\mu_{c}}\left(T, X_{T-t}^{x}\right) & =V(t, x)+\int_{0}^{T-t} \mathrm{E}\left[H^{\mu_{c}}\left(t+v, X_{v}^{x}\right) I\left(X_{v}^{x}>b(t+v)\right)\right] d v \\
& =V(t, x)-\int_{t}^{T} K(t, x, v, b(v)) d v
\end{aligned}
$$


for all $(t, x) \in[0, T) \times(0, \infty)$ where we also use (3.15) and $K$ is defined in (4.1) above. Using (3.11) and (4.9) we see that this yields the representation (4.3). Moreover, since $V(t, b(t))=$ $G^{\mu_{c}}(t, b(t))$ for all $t \in[0, T]$ we see from (4.3) that $b$ solves (4.4). This establishes the existence of the solution to (4.4). We now turn to its uniqueness.

4. We show that the rational exercise boundary is the unique solution to (4.4) in the class of continuous functions $t \mapsto b(t)$ on $[0, T]$ satisfying $b(t) \geq h(t)$ for all $t \in[0, T]$. For this, take any continuous function $c:[0, T] \rightarrow \mathbb{R}$ which solves (4.4) and satisfies $c(t) \geq h(t)$ for all $t \in[0, T]$. Motivated by the representation (4.10) above define the function $U^{c}$ : $[0, T] \times[0, \infty) \rightarrow \mathbb{R}$ by setting

$$
U^{c}(t, x)=\mathrm{E}\left[G^{\mu_{c}}\left(T, X_{T-t}^{x}\right)\right]-\int_{0}^{T-t} \mathrm{E}\left[H^{\mu_{c}}\left(t+v, X_{v}^{x}\right) I\left(X_{v}^{x}>c(t+v)\right)\right] d v
$$

for $(t, x) \in[0, T] \times[0, \infty)$. Observe that $c$ solving (4.4) means exactly that $U^{c}(t, c(t))=$ $G^{\mu_{c}}(t, c(t))$ for all $t \in[0, T]$.

(i) We show that $U^{c}(t, x)=G^{\mu_{c}}(t, x)$ for all $(t, x) \in[0, T] \times[0, \infty)$ such that $x \geq c(t)$. For this, take any such $(t, x)$ and note that the Markov property of $X$ implies that

$$
U^{c}\left(t+s, X_{s}^{x}\right)-\int_{0}^{s} H^{\mu_{c}}\left(t+v, X_{v}^{x}\right) I\left(X_{v}^{x}>c(t+v)\right) d v
$$

is a continuous martingale under $\mathrm{P}$ for $s \in[0, T-t]$. Consider the stopping time

$$
\sigma_{c}=\inf \left\{s \in[0, T-t] \mid X_{s}^{x} \leq c(t+s)\right\}
$$

under $\mathrm{P}$. Since $U^{c}(t, c(t))=G^{\mu_{c}}(t, c(t))$ for all $t \in[0, T]$ and $U^{c}(T, x)=G^{\mu_{c}}(T, x)$ for all $x \geq 0$ we see that $U^{c}\left(t+\sigma_{c}, X_{\sigma_{c}}^{x}\right)=G^{\mu_{c}}\left(t+\sigma_{c}, X_{\sigma_{c}}^{x}\right)$. Replacing $s$ by $\sigma_{c}$ in (4.12), taking $\mathrm{E}$ on both sides and applying the optional sampling theorem, we find that

$$
\begin{aligned}
U^{c}(t, x) & =\mathrm{E}\left[U^{c}\left(t+\sigma_{c}, X_{\sigma_{c}}^{x}\right)\right]-\mathrm{E}\left(\int_{0}^{\sigma_{c}} H^{\mu_{c}}\left(t+v, X_{v}^{x}\right) I\left(X_{v}^{x}>c(t+v)\right) d v\right) \\
& =\mathrm{E}\left[G^{\mu_{c}}\left(t+\sigma_{c}, X_{\sigma_{c}}^{x}\right)\right]-\mathrm{E}\left(\int_{0}^{\sigma_{c}} H^{\mu_{c}}\left(t+v, X_{v}^{x}\right) d v\right)=G^{\mu_{c}}(t, x)
\end{aligned}
$$

where in the last equality we use (3.14). This shows that $U^{c}$ equals $G^{\mu_{c}}$ above $c$ as claimed.

(ii) We show that $U^{c}(t, x) \leq V(t, x)$ for all $(t, x) \in[0, T] \times[0, \infty)$. For this, take any such $(t, x)$ and consider the stopping time

$$
\tau_{c}=\inf \left\{s \in[0, T-t] \mid X_{s}^{x} \geq c(t+s)\right\}
$$

under $\mathrm{P}$. We claim that $U^{c}\left(t+\tau_{c}, X_{\tau_{c}}^{x}\right)=G^{\mu_{c}}\left(t+\tau_{c}, X_{\tau_{c}}^{x}\right)$. Indeed, if $x \geq c(t)$ then $\tau_{c}=0$ so that $U^{c}(t, x)=G^{\mu_{c}}(t, x)$ by (i) above. On the other hand, if $x<c(t)$ then the claim follows since $U^{c}(t, c(t))=G^{\mu_{c}}(t, c(t))$ for all $t \in[0, T]$ and $U^{c}(T, x)=G^{\mu_{c}}(T, x)$ for all $x \geq 0$. Replacing $s$ by $\tau_{c}$ in (4.12), taking $\mathrm{E}$ on both sides and applying the optional sampling theorem, we find that

$$
U^{c}(t, x)=\mathrm{E}\left[U^{c}\left(t+\tau_{c}, X_{\tau_{c}}^{x}\right)\right]-\mathrm{E}\left(\int_{0}^{\tau_{c}} H^{\mu_{c}}\left(t+v, X_{v}^{x}\right) I\left(X_{v}^{x}>c(t+v)\right) d v\right)
$$




$$
=\mathrm{E}\left[G^{\mu_{c}}\left(t+\tau_{c}, X_{\tau_{c}}^{x}\right)\right] \leq V(t, x)
$$

where in the second equality we used the definition of $\tau_{c}$. This shows that $U^{c} \leq V$ as claimed.

(iii) We show that $c(t) \leq b(t)$ for all $t \in[0, T]$. For this, suppose that there exists $t \in[0, T)$ such that $c(t)>b(t)$. Take any $x \geq c(t)$ and consider the stopping time

$$
\sigma_{b}=\inf \left\{s \in[0, T-t] \mid X_{s}^{x} \leq b(t+s)\right\}
$$

under $\mathrm{P}$. Replacing $s$ with $\sigma_{b}$ in (4.6) and (4.12), taking $\mathrm{E}$ on both sides of these identities and applying the optional sampling theorem, we find

$$
\begin{aligned}
& \mathrm{E}\left[V\left(t+\sigma_{b}, X_{\sigma_{b}}^{x}\right)\right]=V(t, x)+\mathrm{E}\left(\int_{0}^{\sigma_{b}} H^{\mu_{c}}\left(t+v, X_{v}^{x}\right) d v\right) \\
& \mathrm{E}\left[U^{c}\left(t+\sigma_{b}, X_{\sigma_{b}}^{x}\right)\right]=U^{c}(t, x)+\mathrm{E}\left(\int_{0}^{\sigma_{b}} H^{\mu_{c}}\left(t+v, X_{v}^{x}\right) I\left(X_{v}^{x}>c(t+v)\right) d v\right) .
\end{aligned}
$$

Since $x \geq c(t)$ we see by (i) above that $U^{c}(t, x)=G^{\mu_{c}}(t, x)=V(t, x)$ where the last equality follows since $x$ lies above $b(t)$. Moreover, by (ii) above we know that $U^{c}\left(t+\sigma_{b}, X_{\sigma_{b}}^{x}\right) \leq$ $V\left(t+\sigma_{b}, X_{\sigma_{b}}^{x}\right)$ so that (4.18) and (4.19) imply that

$$
\mathrm{E}\left(\int_{0}^{\sigma_{b}} H^{\mu_{c}}\left(t+v, X_{v}^{x}\right) I\left(X_{v}^{x} \leq c(t+v)\right) d v\right) \geq 0 .
$$

The fact that $c(t)>b(t)$ and the continuity of the functions $c$ and $b$ imply that there exists $\varepsilon>0$ sufficiently small such that $c(t+v)>b(t+v)$ for all $v \in[0, \varepsilon]$. Consequently the Pprobability of the process $X^{x}=\left(X_{v}^{x}\right)_{0 \leq v \leq \varepsilon}$ spending a strictly positive amount of time (w.r.t. Lebesgue measure) in this set before hitting $b$ is strictly positive. Combined with the fact that $b$ lies above $h$ this forces the expectation in (4.20) to be strictly negative and provides a contradiction. Hence $c \leq b$ as claimed.

(iv) We show that $b(t)=c(t)$ for all $t \in[0, T]$. For this, suppose that there exists $t \in[0, T)$ such that $c(t)<b(t)$. Take any $x \in(c(t), b(t))$ and consider the stopping time

$$
\tau_{b}=\inf \left\{s \in[0, T-t] \mid X_{s}^{x} \geq b(t+s)\right\}
$$

under P. Replacing $s$ with $\tau_{b}$ in (4.6) and (4.12), taking $\mathrm{E}$ on both sides of these identities and applying the optional sampling theorem, we find

$$
\begin{aligned}
& \mathrm{E}\left[V\left(t+\tau_{b}, X_{\tau_{b}}^{x}\right)\right]=V(t, x) \\
& \mathrm{E}\left[U^{c}\left(t+\tau_{b}, X_{\tau_{b}}^{x}\right)\right]=U^{c}(t, x)+\mathrm{E}\left(\int_{0}^{\tau_{b}} H^{\mu_{c}}\left(t+v, X_{v}^{x}\right) I\left(X_{v}^{x}>c(t+v)\right) d v\right) .
\end{aligned}
$$

Since $c \leq b$ by (iii) above and $U^{c}$ equals $G^{\mu_{c}}$ above $c$ by (i) above, we see that $U^{c}(t+$ $\left.\tau_{b}, X_{\tau_{b}}^{x}\right)=G^{\mu_{c}}\left(t+\tau_{b}, X_{\tau_{b}}^{x}\right)=V\left(t+\tau_{b}, X_{\tau_{b}}^{x}\right)$ where the last equality follows since $V$ equals $G^{\mu_{c}}$ above $b$ (recall also that $U^{c}(T, x)=G^{\mu_{c}}(T, x)=V(T, x)$ for all $x \geq 0$ ). Moreover, by (ii) we know that $U^{c} \leq V$ so that (4.22) and (4.23) imply that

$$
\mathrm{E}\left(\int_{0}^{\sigma_{b}} H^{\mu_{c}}\left(t+v, X_{v}^{x}\right) I\left(X_{v}^{x}>c(t+v)\right) d v\right) \geq 0 .
$$


But then as in (iii) above the continuity of the functions $c$ and $b$ combined with the fact that $c$ lies above $h$ forces the expectation in (4.24) to be strictly negative and provides a contradiction. Thus $c=b$ as claimed and the proof is complete.

\section{Financial analysis of the British Asian option}

In the present section we briefly discuss the rational exercise strategy of the British Asian option, and then a numerical example is presented to highlight the practical features of the option. In particular we expect these features to be robust and extend to the other types of British Asian option considered in the following section (or at least provide a useful indication). We draw comparisons with the European Asian option since this option is widely traded (in fact the most traded exotic option) and well understood. In the financial analysis of the option returns presented below we mainly address the question as to what the return would be if the underlying process enters the given region at a given time. Such an analysis may be viewed as a 'skeleton analysis' (i.e. we do not discuss the probability of the latter event nor do we account for any risk associated with its occurrence). Such a 'skeleton analysis' is both natural and practical since it places the question of probabilities and risk under the subjective assessment of the option holder (irrespective of whether the stock price model is correct or not).

1. In Section 3 above we saw that the rational exercise strategy of the British Asian option (the optimal stopping time (3.17) in the problem (3.12) above) changes as one varies the contract drift $\mu_{c}$. This is illustrated in Figure 1 above. We recall that one sets $\mu_{c}<0$. Indeed, if one sets $\mu_{c} \geq 0$ then it is always optimal to stop at once in (3.12). In this case the buyer is overprotected. On the other hand, when $\mu_{c} \downarrow-\infty$ then $b$ virtually disappears in the limit so that it is hardly ever optimal to stop before the time of maturity $T$ and the British Asian option effectively reduces to the European Asian option (unless $r$ or $T$ are unrealistically large). In the latter case a contract drift of $-\infty$ represents an infinite tolerance of unfavourable drifts and the British Asian holder is very unlikely to exercise the option rationally before $T$.

2. In the numerical example below (see Figure 2) the parameter values have been chosen to present the practical features of the British Asian option in a fair and representative way. Assuming that the initial stock price equals 1 , the (arbitrage-free) price of the British Asian option with a contract drift of $\mu_{c}=-0.1$ is 0.976 and the price of the (arithmetic average) European Asian option (with no strike) is 0.952 . We note that the price of the British Asian option with a contract drift of $\mu_{c}=-0.05$ is 0.984 . Observe that the closer the contract drift gets to 0 , the stronger the protection feature provided (with generally better returns), and the more expensive the British Asian option becomes. Recall also that when $\mu_{c} \downarrow-\infty$ then in the limit it is hardly ever rational to exercise before the time of maturity and the price of the British Asian option effectively reduces to the price of the European Asian option. The fact that the price of the British Asian option is very close to the price of the European Asian option is of considerable practical value.

3. Figure 2 shows the returns that the British Asian holder can extract upon exercising his option (in any contingency). Also shown are the returns observed upon selling the European Asian option (at the arbitrage-free price) in the same contingency. This is motivated by the fact that in practice the European option holder may choose to sell his option at any time during 


\begin{tabular}{|l|c|c|c|c|c|c|c|}
\hline Exercise/Selling time (months) & 0 & 2 & 4 & 6 & 8 & 10 & 12 \\
\hline \hline Exercise at 1.4 with $\mu_{c}=-0.1$ & $241 \%$ & $225 \%$ & $210 \%$ & $194 \%$ & $177 \%$ & $160 \%$ & $144 \%$ \\
\hline Selling at 1.4 (European) & $233 \%$ & $219 \%$ & $205 \%$ & $191 \%$ & $177 \%$ & $162 \%$ & $147 \%$ \\
\hline \hline Exercise at 1.2 with $\mu_{c}=-0.1$ & $221 \%$ & $205 \%$ & $189 \%$ & $173 \%$ & $157 \%$ & $140 \%$ & $123 \%$ \\
\hline Selling at 1.2 (European) & $214 \%$ & $200 \%$ & $186 \%$ & $171 \%$ & $156 \%$ & $141 \%$ & $126 \%$ \\
\hline \hline Exercise at 1.0 with $\mu_{c}=-0.1$ & $200 \%$ & $184 \%$ & $169 \%$ & $153 \%$ & $136 \%$ & $119 \%$ & $103 \%$ \\
\hline Selling at 1.0 (European) & $195 \%$ & $181 \%$ & $166 \%$ & $151 \%$ & $136 \%$ & $121 \%$ & $105 \%$ \\
\hline \hline Exercise at $b$ with $\mu_{c}=-0.1$ & $199 \%$ & $167 \%$ & $134 \%$ & $101 \%$ & $68 \%$ & $34 \%$ & $0 \%$ \\
\hline Selling at $b$ (European) & $194 \%$ & $164 \%$ & $133 \%$ & $101 \%$ & $68 \%$ & $35 \%$ & $0 \%$ \\
\hline \hline Exercise at 0.8 with $\mu_{c}=-0.1$ & $180 \%$ & $164 \%$ & $148 \%$ & $132 \%$ & $116 \%$ & $99 \%$ & $82 \%$ \\
\hline Selling at 0.8 (European) & $176 \%$ & $161 \%$ & $146 \%$ & $131 \%$ & $116 \%$ & $100 \%$ & $84 \%$ \\
\hline \hline Exercise at 0.6 with $\mu_{c}=-0.1$ & $159 \%$ & $143 \%$ & $128 \%$ & $112 \%$ & $95 \%$ & $78 \%$ & $62 \%$ \\
\hline Selling at 0.6 (European) & $157 \%$ & $142 \%$ & $127 \%$ & $111 \%$ & $95 \%$ & $79 \%$ & $63 \%$ \\
\hline \hline Exercise at 0.4 with $\mu_{c}=-0.1$ & $139 \%$ & $123 \%$ & $107 \%$ & $91 \%$ & $75 \%$ & $58 \%$ & $41 \%$ \\
\hline Selling at 0.4 (European) & $138 \%$ & $123 \%$ & $107 \%$ & $91 \%$ & $75 \%$ & $59 \%$ & $42 \%$ \\
\hline \hline Exercise at 0.2 with $\mu_{c}=-0.1$ & $118 \%$ & $102 \%$ & $87 \%$ & $70 \%$ & $54 \%$ & $37 \%$ & $21 \%$ \\
\hline Selling at 0.2 (European) & $119 \%$ & $103 \%$ & $87 \%$ & $71 \%$ & $55 \%$ & $38 \%$ & $21 \%$ \\
\hline
\end{tabular}

Figure 2. Returns observed upon exercising the British Asian option compared with returns observed upon selling the European Asian option. The values in the left-hand column represent the position of the process $X=I / S$. The returns are expressed as a percentage of the original option price paid by the buyer (rounded to the nearest integer), i.e. $R(t, x) / 100=G^{\mu_{c}}(t, x) / V(0,0)$ and $R_{E}(t, x) / 100=V_{E}(t, x) / V_{E}(0,0)$ respectively. The parameter set is $T=1, r=0.1, \sigma=0.4$ and the contract drift $\mu_{c}$ equals -0.1 so that $b(0)=0.99$ (recall Figure 1 above). The actual returns depend explicitly upon the stock price $S_{t}$ in relation to its initial value $S_{0}$ and can be recovered by multiplying the tabulated returns by $S_{t} / S_{0}$. The returns shown are sufficient for comparative purposes.

the term of the contract, and in this case one may view his 'payoff' as the price he receives upon selling. Upon inspection of Figure 2 we see that exercising the British Asian option produces remarkably similar returns to selling the European Asian option (at the arbitrage-free price). In particular we observe that this is generally true for every possible stock price path (i.e. every quadruple $\left(t, X_{t}, S_{t}, S_{0}\right)$ using the notation of Section 3 above) and as such whether stock price movements are favourable or unfavourable (independently of any subjective interpretation). However, as remarked in [7] and [8], in a real financial market the option holder's ability and/or desire to sell his contract will depend upon a number of exogenous factors. These include his ability to access the option market, the transaction costs and/or taxes involved in selling (i.e. friction costs), and in particular the liquidity of the option market itself (which in turn determines the market/liquidation price of the option). In the present setting the latter factor is especially important. Asian options generally trade in over-the-counter markets which have no organised exchange and as such these markets can be very illiquid. Crucially, the protection feature of the British Asian option is intrinsic to it, that is, it is completely endogenous. It is inherent in the payoff function itself (obtained as a consequence of optimal prediction), and as such it is independent of any exogenous factors. From this point of view the British Asian option is a particularly attractive financial instrument. 
4. Finally we highlight a remarkable and peculiar aspect of the British Asian option (also seen in the British put and call options [7] and [8]). Namely, it can be verified that the value function and the payoff function of the British Asian option stay close together in the continuation set. In fact one finds (for this particular choice of contract drift) that the value function stays above the payoff function (both expressed as percentage returns) within a margin of two percent. The tightness of this relationship will be affected by the choice of the contract drift (indeed the closer the contract drift is to 0 the stronger the protection and the tighter the relationship will be). Thus we see that (i) exercising in the continuation set produces a remarkably comparable return to selling the contract at the arbitrage-free price (i.e. in a liquid option market); and (ii) even if the option market is perfectly liquid it may still be more profitable to exercise rather than sell (when the friction costs exceed the margin of two percent for instance). In light of the discussion above it is clear that the former observation becomes especially important in the present setting.

\section{Other British Asian options}

We remarked above that the British Asian option analysed in this paper plays a canonical role among all other possibilities. The purpose of this section is to provide a brief review of other British Asian options as discussed in (i)-(iv) of Section 1.

In the setting of (2.1) and (2.2) consider the arithmetic (stock price) average defined by $A_{T}=\frac{1}{T} \int_{0}^{T} S_{t} d t$ and the geometric (stock price) average defined by $G_{T}=\exp \left(\frac{1}{T} \int_{0}^{T} \log \left(S_{t}\right) d t\right)$ where a maturity time $T>0$ and a strike price $K>0$ are given and fixed. Extending Definition 1 of Section 3 in an obvious manner we obtain the following classification of British Asian options (according to their payoffs):

(6.1) The arithmetic average British Asian call/put option with fixed strike:

$$
\mathrm{E}^{\mu_{c}}\left(\left(A_{T}-K\right)^{+} \mid \mathcal{F}_{\tau}\right) \text { and } \mathrm{E}^{\mu_{c}}\left(\left(K-A_{T}\right)^{+} \mid \mathcal{F}_{\tau}\right)
$$

(6.2) The geometric average British Asian call/put option with fixed strike:

$$
\mathrm{E}^{\mu_{c}}\left(\left(G_{T}-K\right)^{+} \mid \mathcal{F}_{\tau}\right) \text { and } \mathrm{E}^{\mu_{c}}\left(\left(K-G_{T}\right)^{+} \mid \mathcal{F}_{\tau}\right)
$$

(6.3) The arithmetic average British Asian call/put option with floating strike:

$$
\mathrm{E}^{\mu_{c}}\left(\left(S_{T}-A_{T}\right)^{+} \mid \mathcal{F}_{\tau}\right) \text { and } \mathrm{E}^{\mu_{c}}\left(\left(A-S_{T}\right)^{+} \mid \mathcal{F}_{\tau}\right)
$$

(6.4) The geometric average British Asian call/put option with floating strike:

$$
\mathrm{E}^{\mu_{c}}\left(\left(S_{T}-G_{T}\right)^{+} \mid \mathcal{F}_{\tau}\right) \text { and } \mathrm{E}^{\mu_{c}}\left(\left(G_{T}-S_{T}\right)^{+} \mid \mathcal{F}_{\tau}\right)
$$

where $\mu_{c}$ is a contract drift (to be examined/specified). One can also consider the geometric average British Asian option (with no strike) and every option above with a differently weighted average (e.g. exponentially weighted) which is also referred to as a flexible average.

The economic rationale of Section 2 extends to all these cases in a straightforward manner (with the necessary modifications when the strike is floating) and answering the pertinent mathematical/financial questions as in Sections 3-5 above leads to an extensive programme of 
research. It should be noted that most of these problems are inherently three dimensional and no reduction to dimension two appears to be possible.

\section{References}

[1] Du Toit, J. and Peskir, G. (2007). The trap of complacency in predicting the maximum. Ann. Probab. 35 (340-365).

[2] Hansen, A. T. And Jørgensen, P. L. (2000). Analytical valuation of American-style Asian options. Management Sci. 46 (1116-1136).

[3] Ingersoll, J. E. (1987). Theory of Financial Decision Making. Blackwell.

[4] Kramkov, D. O. and Mordecky, E. (1994). Integral option. Theory Probab. Appl. 39 (162-173).

[5] Peskir, G. (2005). A change-of-variable formula with local time on curves. J. Theoret. Probab. 18 (499-535).

[6] Peskir, G. (2006). On the fundamental solution of the Kolmogorov-Shiryaev equation. The Shiryaev Festschrift (Metabief 2005), Springer (535-546).

[7] Peskir, G. and Samee, F. (2008). The British put option. Research Report No. 1, Probab. Statist. Group Manchester (25 pp). To appear in Appl. Math. Finance.

[8] Peskir, G. and Samee, F. (2008). The British call option. Research Report No. 2, Probab. Statist. Group Manchester (25 pp).

[9] Peskir, G. and Uys, N. (2005). On Asian options of American type. Exotic Option Pricing and Advanced Lévy Models (Eindhoven 2004), John Wiley (217-235).

[10] Peskir, G. and Shiryaev, A. N. (2006). Optimal Stopping and Free-Boundary Problems. Lectures in Mathematics, ETH Zürich, Birkhäuser.

[11] Shiryaev, A. N. (1999). Essentials of Stochastic Finance. World Scientific.

[12] Yor, M. (1992). On some exponential functionals of Brownian motion. Adv. Appl. Probab. $24(509-531)$.

Kristoffer Glover

School of Finance and Economics

University of Technology

Sydney NSW 2007

Australia

Kristoffer.Glover@uts.edu.au 
Goran Peskir

School of Mathematics

The University of Manchester

Oxford Road

Manchester M13 9PL

United Kingdom

goran@maths.man.ac.uk

Farman Samee

School of Mathematics

The University of Manchester

Oxford Road

Manchester M13 9PL

United Kingdom

Farman.Samee@postgrad.manchester.ac.uk 